\title{
Designing Context-Aware Adaptive Units of Learning Based on IMS-LD Standard
}

\author{
Sergio Gómez ${ }^{\# 1}$, David Huerva ${ }^{\# 2}$, Carolina Mejía ${ }^{\# 3}$, Silvia Baldiris ${ }^{\# 4}$, Ramón Fabregat ${ }^{\# 5}$ \\ \# Institute of Informatics Applications, Universitat de Girona \\ Spain \\ 'sergiog@eia.udg.edu \\ ${ }^{2}$ dhuervad@eia.udg.edu \\ ${ }^{3}$ carolina@eia.udg.edu \\ ${ }^{4}$ baldiris@eia.udg.edu \\ ramon.fabregat@udg.edu
}

\begin{abstract}
Learning contents adaptation has been a subject of interest in the research area of the adaptive hypermedia systems. Defining which variables and which standards can be considered to model adaptive content delivery processes is one of the main challenges in pedagogical design over e-learning environments. In this paper some specifications, architectures and technologies that can be used in contents adaptation processes considering characteristics of the context are described and a proposal to integrate some of these characteristics in the design of units of learning using adaptation conditions in a structure of IMSLearning Design (IMS-LD) is presented. The key contribution of this work is the generation of instructional designs considering the context, which can be used in Learning Management Systems (LMSs) and diverse mobile devices.
\end{abstract}

\section{INTRODUCTION}

Nowadays, research in e-learning about the development of LMSs is oriented to adaptation processes construction considering different traits of users like: learning profiles, interests, competences, knowledge levels, and others described in [1]. The goal is to define optimal ways that the contents and the services, available in this type of systems, should be presented to students and teachers (main actors of learning and teaching processes respectively).

Several projects have proposed mechanisms to present the contents based on the user learning characteristics and preferences (MASPLANG[2], EU4ALL[3], shaboo[4], escen@ri[5]), although the amount that considers user context characteristics such as access device, browser, connection technology, location of the user, physical conditions of environment, etc., is much smaller (MOBILearn [6], AMLMS [7]).

Nevertheless, to define an optimal way of presentation, it is important to consider the existence of heterogeneous characteristics of users like: interaction preferences, learning purposes, tasks to achieve, mood, etc., which will allow to deem with certainty the success of objectives achievement defined in the learning process.
Moreover, it is important to consider the diversity existing in the characteristics that describe the user context. In [8] the necessity to include the context in the implementation of systems is presented and the characteristics and parameters that describe it are defined: User, Place, Device, Time and Physical environment.

Addressing this heterogeneity amongst the users of LMSs, can generate the development of a diversity of independent solutions that may want to solve the problem since different points of view. Nevertheless, if e-learning standards are not used, this would limit the usability and interoperability and even restrict the implementation of adaptation processes.

At present, different existing organisms (IMS Global Learning Consortium [9], IEEE [10], ARIADNE [11], ADL [12], etc.) in charge of the standards definition have created some specifications that consider: the construction of educative contents, the storage of personal characteristics of the students, the definition of competitions, the generation of evaluation structures and the development of instructional designs, in summary each one of the elements involved in the instructional process. These specifications generally do not adjust to a unique pedagogical model and facilitate the exchange of information between different interested entities: people, institutions or systems.

In this proposal we use the specification of the IEEE-LOM [13] standard to characterize learning resources by means of defining a metadata file that helps describing their properties, and the IMS-LD [14] standard because it defines a structure that let different elements to be considered in the design of pedagogical models over different LMSs.

In Fig. 1 the relationship of the elements participating in an IMS-LD is illustrated. This standard describes this relationship as the structure of a theater play, in which there are several scenarios (executions) with different acts that can be played and that involve different actors with several roles (students or teachers). Also there are activities that actors perform in different environments, using different 


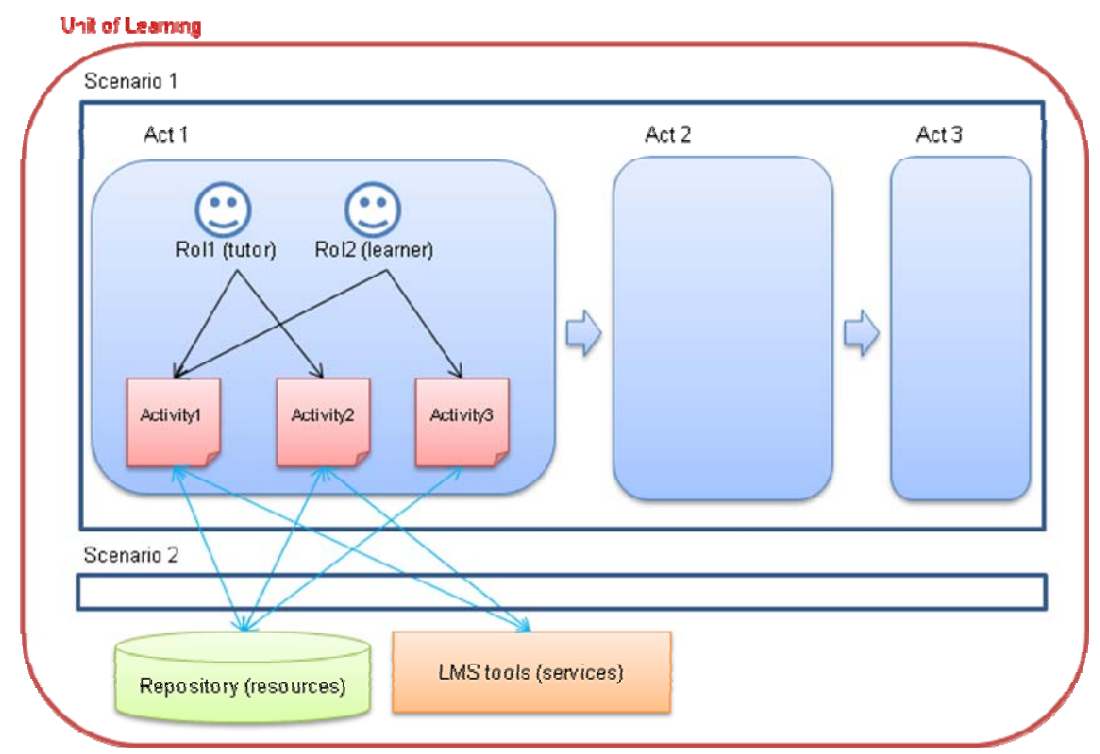

Fig. 1 Relationship of the main elements in an IMS-LD

resources and tools. The result of this structure is a package called "Unit of Learning" (UoL) with specific adaptive properties which are explained in next sections.

The analysis of the context characteristics and the elements that participate in the design of pedagogical models in a LMS, are the focus of this paper by means of proposing a mechanism to build adaptive instructional designs based on the context. This proposal is based on the identification of different user context adaptation variables which are not considered currently in the design of "Units of Learning".

This paper is structured as follows. In the second section the background of the proposal is presented. It describes How the context is conceived and which levels of design can be implemented using the IMS-LD standard. The third section explains some specifications, architectures and technologies used in research to propose context-aware adaptation processes. In fourth section the proposal of a context-aware adaptive design to build an UoL following the IMS-LD standard is presented. Finally some conclusions and future work are remarked.

\section{BACKGROUND}

\section{A. Characteristics of context}

With the increasing use of diverse mobile devices that allow people to access the information anytime and anywhere, the computer science acquired a different perspective marked by Weiser's vision, who introduced the term of ubiquitous computing (ubicomp) [15], which defines a seamlessly integration between systems in the environment that aids people in their everyday activities. Under this concept, new technology has been embedded in many everyday objects and places such as furniture, clothes, museums, theaters, roads, vehicles, etc., to provide people with necessary tools and processes that allow them to do their activities by means of a relaxing interaction with the environment.
Mobile devices are the type of objects with a greater index of usability, because they are devices that join several tools and services that can be accessed at any time. In addition, they satisfy the nomadicity needs that people have when they are moving and frequently carrying everyday tasks to any place.

Nowadays, mobile technology has different and sophisticated types of presentation such as mobile telephones, notebooks, PDA's, tablet PC, among others, which offer a variety of possibilities to access the information and present different technological characteristics. For instance, with ubiquitous computing, integration of this variety and systems are tried to be carried out by means of building applications that any device can use, in order to let the users access the same information using the device of their preference.

Particular technological characteristics of devices and other before mentioned parameters like: user preferences, place properties, access moment, among others, are part of what is denominated as context, that according to [16] can be defined as "any information that can be used to characterize the situation of an entity", understanding the term "entity" as anything relevant participating in the interaction between an user and a system, such as a person, a place, or an object including the user and the system.

In e-learning, this concept is revealed in learning tools development, architecture design, technologies application and specifications definition in order to design and implement processes to adapt contents and services that are displayed to either students in their learning process and teachers in monitoring during their teaching process. The majority of works done in this area have been focused on addressing context characteristics of access devices [17], and other "technological characteristics" such as browser, connection technology, server state, etc. Nevertheless, the term context can consider many other characteristics. In [8], Eleni presents five parameters in which the characteristics of context can be classified (see Table 1). The parameters are: User, Place, Artefact, Time and Physical environment. With these 
parameters an ontology that lets modeling the context in order to build mobile applications was defined.

TABLE I

PARAMETERS AND CHARACTERISTICS OF CONTEXT

\begin{tabular}{|l|l|}
\hline Parameters & Characteristics \\
\hline User & $\begin{array}{l}\text { profile, preferences, goals, tasks, } \\
\text { activities, actions, mood, etc. }\end{array}$ \\
\hline Place & $\begin{array}{l}\text { location, orientation, space (public, } \\
\text { private, social, informative), etc. }\end{array}$ \\
\hline Artefact & $\begin{array}{l}\text { physical properties, digital properties, } \\
\text { interaction interfaces, etc. }\end{array}$ \\
\hline Time & $\begin{array}{l}\text { hour, day, weekday, month, season, year, } \\
\text { etc }\end{array}$ \\
\hline $\begin{array}{l}\text { Physical } \\
\text { environment }\end{array}$ & $\begin{array}{l}\text { weather condition, illumination, noise } \\
\text { level, overcrowding, etc }\end{array}$ \\
\hline
\end{tabular}

Each parameter has different characteristics that can be used to identify adaptation context variables as we made in this proposal.

\section{B. Properties of an IMS-LD unit of learning}

Unit of Learning (UoL) is a term very known in e-learning and is used to refer any delimited educative or training piece like a course, a module, a lesson, etc.

IMS-LD specification is a standard that defines a modeling language to build this UoL. It defines UoL as "the smallest unit providing learning events for learners, satisfying one or more inter-related learning objectives", and provides in [14] the elements that it contains: a manifest file (an XML file that represents the complete structure of the UoL), a instruccional design, the definition of resources, optional sub-manifest and physical files.

The instructional design is the key element in a UoL and is basically what it is tried to be modeled; the other elements are necessary and complementary parts. In literature the term used to refer to instructional design is "learning design". Some definitions that are considered important to highlight according to its meaning are: in [18] MacNeil defines the learning design as "the systematic process of translating general principles of learning and instruction into plans for instructional materials and learning", and according to the specification it means: "a description of a method enabling learners to attain certain learning objectives by performing certain learning activities in a certain order in the context of a certain learning environment".

In order to build a UoL, IMS-LD describes and implements learning activities based on different pedagogical methodologies, including group work and collaborative learning. In addition, it lets to coordinate multiple learners and roles within a model unique-student or multi-student. It let manage the use of learning contents with collaboration services and also it support models delivery including blended learning (face-to-face instruction combined with computermediated instruction).

With the purpose of facilitate the implementation of the specification in different LMS, IMS-LD can be built in three different levels: level A, level $\mathrm{B}$ and level $\mathrm{C}$, providing different schemes of XML for each level that can be integrated considering the purpose wanted for a certain LMS. The level A offers the necessary vocabulary to express a general learning process, including learning paths. It considers the definition of different user roles in the process (e.g. teacher and student), several learning and support activities that these roles perform and diverse environments in order to establish which learning resources and services can be used in those activities. The level $\mathrm{B}$, adds the possibility of defining conditions to evaluate different expressions based on properties of the individual user or the roles in order to enable the personalization. Finally, the level $\mathrm{C}$ allows a notification mechanism between roles to be defined (i.e. messages as answers to events execution).

Our proposal considers level B of the IMS-LD in order to generate adaptive UoL according with the characteristics of the context. This can be achieved defining properties and conditions that let refining the visibility of the activities and environment entities to different users and roles, specifically to their access devices. The use of conditions and properties allow the authors of learning designs to consider different variables of adaptation in order to let them schematize a pedagogical model on a LMS. We consider the proposal made in [19] that integrates different specifications and standards in an IMS-LD. That work describes how can be developed learning designs addressing variables of adaptation according to the learning profile and competitions of the student. Continuing with the capacities of the standard in [20] is presented how the use of conditions can be used to generate level B learning designs to adapt resources according to the student's learning profile.

To conclude, another important characteristic in UoL building is the relation and integration with other specifications like: IMS Content Packaging [21] for distribution of UoLs, the IMS/LOM Metadata [22] for characterization of the resources, IMS Learner Information Package [23] for characterization of the students, among others. This property adds interoperability and durability to the different proposed models in order to obtain adaptive UoLs.

\section{CONTEXT-AWARE ADAPTATION}

Considering the parameters of the context explained in the background section and the categorization of the levels defined in the IMS-LD standard to obtain adaptive UoL, we could define the properties that can be considered to design the adaptation process in learning designs.

In this section we present some mechanisms used in several works that are useful to implement content adaptation processes on a LMS considering characteristics of the context. We want to expose the different existing alternatives, to define methods that aim to propose different solutions to the problem of building adaptive systems that let including variables like the context.

In content adaptation model on a LMS there are two fundamental elements participating: the users and the educative contents. Within an e-learning process, the "users" 
refer specifically to students and teachers that participate actively in that process; and the "contents" refer to learning resources that the users can exploit. These resources in general can be accessed through LMS and they are created following educative and technological guidelines suitable to achieve the learning objectives in a determined pedagogical model.

When the context is considered in this model, it is necessary to include the participation of external variables that can get involved in the interaction between the users and the LMS in particular with the contents. This new model is defined as "context-aware adaptation" and it is used to refer an adaptation process that considers the characteristics of the context-aware computing. According with [24] these characteristics include the use of the context to:

- present information and services to user

- automatically execute a service for user and

- tag information to support later retrieval.

Taking into account these characteristics some works that propose different context-aware adaptation models to display educative contents are presented.

In MAS-SHAAD [17] a multi-agent architecture is presented to capture the characteristics of the user access device and to automatically build HTML pages by means of using XSLT templates adapted according to the capabilities of the device. In MASHA [25] also a multi-agent architecture is defined, which collect information of the user behavior, when she accesses web pages using different mobile devices, in order to create a global profile that let recommend web pages adapted according to the analysis of that profile or other profiles of users who have used the same access devices. In [26] a functional architecture based on adaptation algorithms let to present customized intelligent contents to the characteristics of the access devices.

To obtain the access device properties these works and other context-aware proposal systems applied different specifications such as CC/PP [27], UAProf [28] and WURLF [29], which defines the profiles of the devices in a XML structure that has data related to their technological capabilities (hardware, software, network, etc.). Also, some of these proposals use repositories that are created to allow these profiles to be stored in order to use them in the implementation of systems that consider the context. Two examples of these repositories are SESAME [30] that stores UAProf profiles and the repository of WURLF that stores WURLF profiles respectively.

Other mechanisms to adapt contents that are widely used are the HTML Mobile transcoders, which let personalize the file format or the properties of the resources according to generated requests of modification. Some of these mechanisms are used in Phonifier [31], IYHY.com [32], and others. They are available in web sites and can be integrated to any architecture because they are independent methods that can be used through HTTP requests.

In conclusion, there are different existing proposed mechanisms that model content adaptation process based on the context, exclusively on the technological capabilities of the devices.
Taking into account the information explained, we present a proposal to design a unit of learning that leaves the free possibility to integrate mechanisms, specifications and technologies of adaptation, like the ones we discussed in this section. But that can be considered after some set of conditions based on the general characteristics of the context are specified.

\section{DESIGN OF A CONTEXT-AWARE ADAPTIVE IMS-LD}

In order to carry out the context-aware adaptation design, the work done in [33] was considered as preliminary work of this proposal. It defines a process to adapt the learning resources according to the technological characteristics of the mobile access device, which means, that it considered as first scope some characteristics of the parameter "artefact" of the context.

Considering that, the context-aware adaptation design proposed includes three elements: i) the data corresponding to the characteristics of the context, ii) the characteristics of the learning resources stored in a repository and iii) the adaptation conditions that relates the information between i) and ii) in a IMS-LD within a LMS. In Fig. 2 the relationship of the three elements is illustrated.

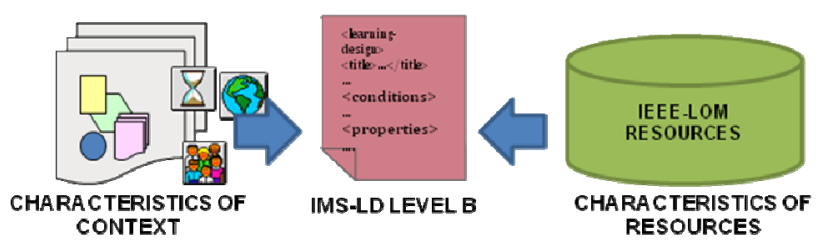

Fig 2 Relationship between the characteristics of the context and the learning resources in an IMS-LD.

In [33] some technological device characteristics were selected for the adaptation process and from that selection those characteristics were considered to propose the conditions presented. These characteristics are listed in Table 2.

TABLE II

Technological CHaRacteristics of the Mobile DeVices

\begin{tabular}{|l|}
\hline Hardware \\
\hline Capacity to show colors \\
Capacity to show images \\
Capacity to reproduce sounds \\
\hline Software \\
\hline Types (MIME) of the software allowed \\
Capacity to execute Java applications \\
Java Platforms supported \\
\hline Network \\
\hline Capacity to show frames \\
Capacity to show tables \\
Capacity to show Java Applets \\
Capacity to execute Javascript \\
\hline
\end{tabular}

Taking into account the conditions and properties mechanism that can be used to build a UoL is possible to 
determine what learning activities, resources or services will be presented to the student.

To adequate the conditions in the IMS-LD, it was necessary to label resources available in the LMS using metadata. We use IEEE-LOM standard to characterize resources, defining the most important elements of the metadata. These resources are stored in a content repository and are created by teachers, designers and developers, who also have to label following the specification guidelines. The repository used to store contents is Fedora [34]. This repository stores digital resources and let associate metadata of different specifications to them, establish relations between resources and define multiples versions of one content.

The disposition of different versions from a same resource is considered because these could be differentiated and be catalogued individually using the properties of repository and will be available to establish the relations in the conditions with the characteristics of the context.

The conditions consist of sentences IF/THEN/ELSE. Within of IF block an expression that must generate a boolean result is evaluated, in order to execute the adaptation actions within THEN block when the result is true or the actions of ELSE block when the result is false.

Table 3 presents a pseudo-code illustrating an example of conditions that can be applied to the structure of a IMS-LD.

TABLE III

EXAMPLE OF PSEUdO-CODE OF THE CONDITIONS STRUCTURE

\begin{tabular}{|l|l|}
\hline Versions & Conditions \\
\hline \multirow{4}{*}{ V1 } & $\begin{array}{l}\text { if ResolutionX }>\text { CA_V1_ResolutionX then } \\
\text { Score_CA_V1 }=\text { Score_CA_V1 }+1 \text { else } \\
\text { Score_CA_V1 }-1\end{array}$ \\
& $\begin{array}{l}\text { if ResolutionY }>\text { CA_V1_ResolutionY then } \\
\text { Score_CA_V1 }=\text { Score_CA_V1 }+1 \text { else } \\
\text { Score_CA_V1 }-1\end{array}$ \\
\hline & $\begin{array}{l}\text { if ResolutionX }>\text { CA_V2_ResolutionX then } \\
\text { Score_CA_V2 }=\text { Score_CA_V2 }+1 \text { else }\end{array}$ \\
& $\begin{array}{l}\text { Score_CA_V2 }-1 \\
\text { if ResolutionY }>\text { CA_V2_ResolutionY then }\end{array}$ \\
& $\begin{array}{l}\text { Score_CA_V2 }=\text { Score_CA_V2 }+1 \text { else } \\
\text { Score_CA_V2 }-1\end{array}$ \\
\hline \multirow{5}{*}{ V3 } & $\begin{array}{l}\text { if ResolutionX }>\text { CA_V3_ResolutionX then } \\
\text { Score_CA_V3 }=\text { Score_CA_V3 }+1 \text { else } \\
\text { Score_CA_V3 }-1 \\
\text { if ResolutionY }>\text { CA_V3_ResolutionY then }\end{array}$ \\
& $\begin{array}{l}\text { Score_CA_V3 }=\text { Score_CA_V3 }+1 \text { else } \\
\text { Score_CA_V3 }-1\end{array}$ \\
\hline
\end{tabular}

In table 3 is shown how our proposal uses different versions from the resources (V1, V2 and V3) to determine the adaptation according to the characteristics of the context. Each row represents the conditions for a version from a same resource. Conditions generated here make reference to the technological properties, specifically to the capabilities of hardware. If the resolution size of the mobile screen on $\mathrm{x}$-axis is greater than the resource size in the same axis, then a score variable will be increased in 1 , otherwise it is decreased in 1 . In the developed work we took the result from the satisfaction of these conditions like a total score for each one of the versions of the resource, which will let the system to select the resource that better fits to the characteristics of the device.

In order to get the adaptation in a Unit of Learning considering the parameters of the context, it will be necessary to define a set of conditions like the shown in the Table 3 that allow to evaluate different characteristics that describe the context and in this way may decide what resources will be presented to the user.

\section{CONCLUSIONS AND FUTURE WORKS}

The contribution of the work is the generation of adaptive UoL considering different context characteristics that can be used in different LMSs and several access devices.

A proposal that allows elements of the adaptive hypermedia systems area and ubiquitous computing area to be integrated is presented, that through the construction of learning designs can consider adapting variables from the "parameters of context." Ubiquitous computing is an area that aims proposing solutions to situations and problems that had not been considered in the construction of systems, such as contextawareness.

To carry out the context-aware adaptation of an LMS, capturing technological characteristics of the "artefact" is presented as a first scope. It is considered as future work to identify more characteristics of the parameters of context (user, place, artefact, time, physical environment) that can be used in the construction of a learning design.

Furthermore, the use of mechanisms to generate modifications to educational content is presented and considered to implement content adaptation mechanisms after the conditions have been processed. We concluded that using them can facilitate the integration of an adaptation process to any architecture. Furthermore, they can aim to implement adaptation in run-time process when an IMS-LD that considers context is being played. Also to generate different versions of a resource that later can be stored in a repository.

In the construction of an IMS-LD the fact of defining a condition for each characteristic from mobile capabilities and resources, can result in an overly complex and tedious task, which also should not be made by a user. As future work is expected to develop an automated process that generates a set of conditions to be applied in the structure of an IMS-LD.

\section{ACKNOWLEDGMENT}

Authors would like to thank the Spanish Science and Education Ministry for the financial support of A2UN@ project (TIN2008-06862-C04-02/TSI). Also thanks to the Programme Alban, the European Union Programme of High Level Scholarships for Latin America, scholarship No. E06D103680CO and the Research Program of the University of Girona.

\section{REFERENCES}

[1] P. Brusilovky and E. Millán, "User Model for Adaptive Hypermedia and Adaptive Educational Systems", The Adaptive Web, LNCS 4321, P. Brusilovsky, A. Kobsa, and W. Nejdl, Eds. Berlin, Germany: SpringerVerlag, pp. 3-53, 2007. 
[2] C. I. Peña, "Intelligent agents to improve adaptivity in a web-based learning environment", PhD Thesis, Universitat de Girona, Girona, Spain, 2004.

[3] O.C. Santos and J.G. Boticario, 'European unified approach for accessible lifelong learning', in A. Méndez-Vilas et al. (Eds.) Current Developments in Technology-Assisted Education, Vol. II, pp. 1102$1106,2006$.

[4] G. D. Moreno and S. M. Baldiris, "Sistema Hipermedia Adaptativo para la Enseñanza de la Programación Orientada a Objetos", Universidad Industrial de Santander, Bucaramanga, Colombia, 2003.

[5] C. Peña, S. Gómez, C. Mejía, and R. Fabregat, "The ProSPETIC Project: A Pioneer Latin-American Initiative for Higher Education Using Information and Communication Technologies", in International Conference of Education, Research and Innovation, IBSN: 978-84612-5091-2, Spain, 2008.

[6] J. Taylor, G. Bo, R. Bernazzani and M. Sharples, "MOBIlearn WP4 Best practices for instructional design and content development for mobile learning", The Open University, Netherlands, MOBIlearn Tech. Rep. D4.2/1.0, 2005.

[7] H. Park, Y. K. Baek and D. Gibson, "Design of an Adaptive Mobile Learning Management System", User interface design and evaluation for mobile technology, vol I, pp. 286-301, 2008.

[8] E. Chirtopoulou, "Context as a Necessity in Mobile Applications", User interface design and evaluation for mobile technology, vol I, pp. 187-204, 2008.

[9] (2009) The IMS Global Learning Consortium website. [Online]. Available: http://www.imsglobal.org/.

[10] (2009) The IEEE website. [Online]. Available: http://www.ieee.org/portal/site.

[11] (2009) The ARIADNE website. [Online]. Available: http://www.ariadne-eu.org/.

[12] (2009) The ADL Advanced Distributed Learning website. [Online]. Available: http://www.adlnet.gov/index.cfm.

[13] Standard for Learning Object Metadata (LOM), IEEE 1484.12.1, 2002.

[14] Learning Design (LD) Specification, IMS Global Learning Consortium, Version 1.0 Final Specification, 2003.

[15] M. Weiser, "The computing for the 21st century". Scientific American, 265 (3), pp. 94-104, 1991.

[16] A. K. Dey and G. D. Abowd, "Towards a better understanding of context and context-awareness" in CHI 2000 Workshop on The What, Who, Where, When, Why and How of Context-awareness, pp.1-6, 2000.

[17] D. Mérida, M. Cannataro, R. Fabregat and C. Arteaga, "MAS-SHAAD a Multiagent System Proposal for an Adaptive Hypermedia System", in Proceedings of IJCEELL journal Special issue: Adaptivity in Web and Mobile Learning Services, 2004
[18] S. McNeil. (2008) Instruccional Design - Course of Masters Degree in Instructional Technology Program webpage on University of Huston. [Online]. Available: http://www.coe.uh.edu/courses/cuin6373/index.html.

[19] S. Baldiris, O. C. Santos, C. Barrera, J. G. Boticario, J. Velez and R. Fabregat, "Integration of educational specifications and standards to support adaptive learning scenarios in ADAPTAPlan", International Journal of Computer Science and Applications (IJCSA), Special Issue on New Trends on AI techniques for Educational Technologies, Vol. 5 , No.1., pp. 88-107, 2008

[20] C. Mejía, S. Baldiris, S. Gómez and R. Fabregat, "Personalization of ELearning Platforms Based On an Adaptation Process Supported on IMS-LIP and IMS-LD", in SITE2009 Society for Information Technology \& Teacher Education, Charleston, 2009.

[21] Content Packaging (CP) Specification, IMS Global Learning Consortium, Version 1.1.4 final specification, 2004.

[22] Metadata (MD) Specification, IMS Global Learning Consortium, Version 1.2.1, 2001

[23] Learner Information Package (LIP) Specification, IMS Global Learning Consortium, Version 1.0 Final Specification, 2001.

[24] A. K. Dey and J. Häkkilä, "Context-Awareness and Mobile Devices", User interface design and evaluation for mobile technology, vol I, pp. 205-217, 2008.

[25] R. Domenico and M. L. Giuseppe, "MASHA: A multi-agent system handling user and device adaptivity of Web sites", Science Business Media B.V., Ed. Berlin, Germany: Springer, pp. 435-462, 2006.

[26] Z. Xinyou, A. Fumihiko, N. Toshie and O. Toshio, "Personalized Adaptive Content System for Context-Aware Mobile Learning", IJCSNS International Journal of Computer Science and Network Security, vol. 8, pp. 153-161, 2008.

[27] CC/PP. (2007) Composite capabilities/preferences profile homepage on W3C. [Online]. Available: http://www.w3.org/Mobile/CCPP/.

[28] User Agent Profile (UAProf) Specification, Wapforum, UAProf19991110, 2008.

[29] (2009) The WURFL website. [Online]. Available: http://wurfl.sourceforge.net/.

[30] (2009) The OpenRDF website. [Online]. Available:

[31] (2008) The Phonifier website. [Online]. Available: http://www.phonifier.com

[32] (2009) The IYHY.com website. [Online]. Available: http://iyhy.com/

[33] D. Huerva, "Integració del sistema MAS-SHAAD amb la plataforma dotLRN", M. Eng. thesis, Universitat de Girona, Girona, Spain, Jul. 2008

[34] (2008) The FedoraCommons website. [Online]. Available: http://www.fedora.info/ 